\title{
Effective Hamiltonian and excitation spectrum of harmonically trapped bosons
}

\author{
Andrij Rovenchak \\ Department for Theoretical Physics, Ivan Franko National University of Lviv \\ 12 Drahomanov St., Lviv 79005, Ukraine \\ E-mail: andrij.rovenchak@gmail.com
}

Received April 3, 2015, revised July 31, 2015, published online November 23, 2015

\begin{abstract}
An approach is proposed to obtain an effective Hamiltonian of a harmonically trapped Bose-system. Such a Hamiltonian is quadratic in the creation-annihilation operators and certain approximations allow to simplify higher (three and four operator) products to the required form. After the Hamiltonian diagonalization, the expression for the excitation spectrum is obtained containing in particular temperature-dependent corrections. Numerical calculations are made for a one-dimensional system. Some prospects towards the extension of the suggested approach to study binary bosonic mixtures are briefly discussed.
\end{abstract}

PACS: 03.75.-b Matter waves;

05.30.-d Quantum statistical mechanics;

67.85.-d Ultracold gases, trapped gases.

Keywords: Bose-system, excitation spectrum, harmonic traps, approximate second quantization.

\section{Introduction}

After the discovery of the Bose-Einstein condensation (BEC) in ultracold alkali gases [1,2], studies of trapped dilute bosonic systems received a new impulse. The issue of collective excitations in such systems appeared in the focus of research immediately [3-5]

$\mathrm{BEC}$ in harmonic traps was discussed in particular in [6-8], with quasi-one-dimensional systems studied in [9]. Reviews on relevant quantum many-body phenomena from both experimental and theoretical points of view can be found, for instance, in [10-13].

A description of excitations in trapped bosonic systems beyond mean-field or Gross-Pitaevskii approximations cannot be considered a completely solved problem at present. Studies of excitation spectra of low-dimensional trapped bosonic systems was made, e.g., in [14,15] using also the Lieb-Liniger theory. In the analysis of Bosecondensates in optical lattices the Bose-Hubbard model is often applied [16-18]. Mathematical aspects of the Bogoliubov approximation [19] for excitation spectra of weakly-interacting bosons are discussed in [20,21]. Theoretical description of Bogoliubov-type excitations in exciton-polariton Bose-condensates is given in [22].

Another possibility to study a trapped Bose-system using the second quantization approach is addressed in this work. Certain approximations are suggested to take into consideration items in the Hamiltonian beyond quadratic in the creation-annihilation operators. The obtained effective excitation spectrum contains in particular temperaturedependent corrections.

Such an approach correlates with the Hartree-FockBogoliubov (HFB) approximation and its extensions. A gapeless modification was proposed in [23] for homogenous Bose-condensates. Analysis of transport properties of Bose-condensates in mesoscopic waveguides was given in [24]. Bosons in random potentials were studied in [25], see also [26] for a comprehensive review containing, in particular, analysis of the HFB approximation in the nonuniform case and its generalizations. The density-functional theory to study Bose-condensates at finite temperatures was applied in [27]. Thermodynamic properties of a Bose-gas beyond the HFB approximation were analyzed in [28] using imaginary-time Green's functions. Hard-sphere bosonic systems were considered within a self-consistent $t$-matrix theory in the HFB approximation [29] and beyond [30]. Recently, the HFB approximation was applied to study phase separation in two-component Bose-condensates [31]. This approximation was also used to describe a finiteparticle trapped one-dimensional Bose-system [32].

The main idea of the present work is to suggest a mathematically simple approximation to account for higherorder corrections in the Hamiltonian. This is done in a phenomenological way by linking creation-annihilation 
operator products to the respective occupation numbers. The obtained analytical expressions might be further also used with results of more thorough derivations.

The paper is organized as follows. In the next Section, the Hamiltonian of the problem is written in the second quantization formalism with some simplification made to account for a macroscopic occupation of the ground state. The diagonalization procedure leading to an effective Hamiltonian is described in Sec. 3. Numerical analysis of a one-dimensional problem is given in Sec. 4. Some prospects of the proposed techniques in application to binary bosonic mixtures are analyzed in Sec. 5. A brief discussion in Sec. 6 concludes the paper.

\section{Hamiltonian in the second quantization formalism}

Let a $D$-dimensional system of $N$ weakly-interacting bosons of mass $m$ be confined to a harmonic trap

$$
V\left(x_{1}, \ldots, x_{D}\right)=\frac{m}{2}\left(\omega_{1}^{2} x_{1}^{2}+\ldots+\omega_{D}^{2} x_{D}^{2}\right)
$$

and the potential of interatomic interaction is given by

$$
U\left(x_{1}, \ldots, x_{D}\right)=g \delta(\mathbf{x}),
$$

where the vector $\mathbf{x}=\left(x_{1}, \ldots, x_{D}\right)$ and $g$ is the coupling constant.

The Hamiltonian of such a system reads:

$$
\hat{H}=\sum_{i=1}^{N}\left[\frac{\hat{\mathbf{p}}_{i}^{2}}{2 m}+V\left(\mathbf{x}_{i}\right)\right]+\sum_{1 \leq i<j \leq N} U\left(\mathbf{x}_{i}-\mathbf{x}_{j}\right)=\hat{H}_{0}+\hat{U}
$$

Here, $\hat{\mathbf{p}}_{i}$ is the momentum operator of the $i$ th particle, $x_{i}$ is its coordinate.

It is possible to develop the second quantization approach using the eigenfunctions of the operator $\hat{H}_{0}$, i. e., an ordinary $D$-dimensional harmonic oscillator.

Let $\hat{a}_{j}^{\dagger}, \hat{a}_{j}$ be the creation and annihilation operators for the state $|\mathbf{j}\rangle=\left|j_{1}, \ldots, j_{D}\right\rangle$, respectively. The corresponding energy levels

$$
\varepsilon_{\mathbf{j}}=\hbar\left(\omega_{1} j_{1}+\ldots+\omega_{D} j_{D}\right)
$$

In this representation the Hamiltonian is

$$
\hat{H}=\sum_{\mathbf{j}} \varepsilon_{\mathbf{j}} \hat{a}_{\mathbf{j}}^{\dagger} \hat{a} \mathbf{j}+\frac{1}{2} \sum_{\mathbf{j}, \mathbf{k}, \mathbf{l}, \mathbf{m}}\langle\mathbf{j} \mathbf{k}|U| \mathbf{l m}\rangle \hat{a}_{\mathbf{j}}^{\dagger} \hat{a}_{\mathbf{k}}^{\dagger} \hat{a}_{\mathbf{l}} \hat{a}_{\mathbf{m}}
$$

The operators satisfy standard bosonic commutation relations:

$$
\left[\hat{a}_{\mathbf{j}}, \hat{a}_{\mathbf{k}}^{\dagger}\right]=\delta_{\mathbf{j k}} \text {. }
$$

Following Bogoliubov [19], we assume the occupation number of the lowest state $N_{0}$ is a macroscopic number. As it is an eigenvalue of the operator $\hat{a}_{0}^{\dagger} \hat{a}_{0}$, one can treat $\hat{a}_{0}^{\dagger}$ and $\hat{a}_{0}$ as $c$-numbers, so that

$$
\begin{gathered}
\hat{a}_{0}^{\dagger} \hat{a}_{0}=N_{0}, \quad \hat{a}_{0} \hat{a}_{0}^{\dagger}=N_{0}+1 \simeq N_{0}, \\
\hat{a}_{0}^{\dagger} \simeq \sqrt{N_{0}}, \quad \hat{a}_{0} \simeq \sqrt{N_{0}} .
\end{gathered}
$$

Upon singling out items with one, two, three, and four operators with the zero index, the Hamiltonian becomes:

$$
\begin{aligned}
\hat{H} & =\text { const }+\sum_{\mathbf{j} \neq 0} \varepsilon_{\mathbf{j}} \hat{a}_{\mathbf{j}}^{\dagger} \hat{a}_{\mathbf{j}}+N_{0}^{3 / 2} \sum_{\mathbf{j} \neq 0}\left\{\langle\mathbf{j} 0|U| 00\rangle \hat{a}_{\mathbf{j}}^{\dagger}+\langle 00|U| \mathbf{j} 0\rangle \hat{a}_{\mathbf{j}}\right\}+\frac{N_{0}}{2} \sum_{\mathbf{j}, \mathbf{k} \neq 0}\left\{4\langle\mathbf{j} 0|U| \mathbf{k} 0\rangle \hat{a}_{\mathbf{j}}^{\dagger} \hat{a}_{\mathbf{k}}+\langle\mathbf{j k}|U| 00\rangle \hat{a}_{\mathbf{j}}^{\dagger} \hat{a}_{\mathbf{k}}^{\dagger}+\right. \\
& \left.+\langle 00|U| \mathbf{j} \mathbf{k}\rangle \hat{a}_{\mathbf{j}} \hat{a}_{\mathbf{k}}\right\}+\frac{N_{0}^{1 / 2}}{2} \sum_{\mathbf{j}, \mathbf{k}, \mathbf{l} \neq 0}\left\{2\langle\mathbf{j k}|U| \mathbf{1 0} 0\rangle \hat{a}_{\mathbf{j}}^{\dagger} \hat{a}_{\mathbf{k}}^{\dagger} \hat{a}_{\mathbf{l}}+2\langle\mathbf{j} 0|U| \mathbf{k} \mathbf{\mathbf { l }}\rangle \hat{a}_{\mathbf{j}}^{\dagger} \hat{a}_{\mathbf{k}} \hat{a}_{\mathbf{l}}\right\}+\frac{1}{2} \sum_{\mathbf{j}, \mathbf{k}, \mathbf{l}, \mathbf{m} \neq 0}\langle\mathbf{j k}|U| \mathbf{I m}\rangle \hat{a}_{\mathbf{j}}^{\dagger} \hat{a}_{\mathbf{k}}^{\dagger} \hat{a}_{\mathbf{l}} \hat{a}_{\mathbf{m}},
\end{aligned}
$$

where "const" denotes items of a non-operator nature. We will not neglect the terms having more than two operators with non-zero index as it is usually done in the approximate second quantization, but instead will further try to take them into account in some effective manner.

It was shown in [33] that items linear in $\hat{a}^{\dagger}$ or $\hat{a}$ produce solely a constant shift of the energy levels, so one can neglect them in further analysis. Dropping also a constant term, we obtain the following Hamiltonian:

$$
\begin{gathered}
\hat{H}=\sum_{\mathbf{j} \neq 0} \varepsilon_{\mathbf{j}} \hat{a}_{\mathbf{j}}^{\dagger} \hat{a}_{\mathbf{j}}+\frac{N_{0}}{2} \sum_{\mathbf{j}, \mathbf{k} \neq 0} c_{\mathbf{j} \mathbf{k}}\left(4 \hat{a}_{\mathbf{j}}^{\dagger} \hat{a}_{\mathbf{k}}+\hat{a}_{\mathbf{j}}^{\dagger} \hat{a}_{\mathbf{k}}^{\dagger}+\hat{a}_{\mathbf{j}} \hat{a}_{\mathbf{k}}\right)+ \\
+N_{0}^{1 / 2} \sum_{\mathbf{j}, \mathbf{k}, \mathbf{l} \neq 0} c_{\mathbf{j} \mathbf{k} \mathbf{l}}\left(\hat{a}_{\mathbf{j}}^{\dagger} \hat{a}_{\mathbf{k}}^{\dagger} \hat{a}_{\mathbf{l}}+\hat{a}_{\mathbf{j}}^{\dagger} \hat{a}_{\mathbf{k}} \hat{a}_{\mathbf{l}}\right)+ \\
+\frac{1}{2} \sum_{\mathbf{j}, \mathbf{k}, \mathbf{l}, \mathbf{m} \neq 0} c_{\mathbf{j} \mathbf{k} \mathbf{l} \mathbf{m}} \hat{a}_{\mathbf{j}}^{\dagger} \hat{a}_{\mathbf{k}}^{\dagger} \hat{a}_{\mathbf{l}} \hat{a}_{\mathbf{m}},
\end{gathered}
$$

where, recalling that the harmonic oscillator eigenfunctions are real-valued,

$$
\begin{gathered}
c_{\mathbf{j k}}=\langle\mathbf{j} 0|U| \mathbf{k} 0\rangle=\langle\mathbf{j k}|U| 00\rangle=\langle 00|U| \mathbf{j k}\rangle, \\
c_{\mathbf{j k l}}=\langle\mathbf{j k}|U| \mathbf{l} 0\rangle=\langle\mathbf{j} 0|U| \mathbf{k} \mathbf{}\rangle, \\
c_{\mathbf{j k} \mathbf{l m}}=\langle\mathbf{j k}|U| \mathbf{l m}\rangle .
\end{gathered}
$$

\section{Diagonalization procedure}

In order to obtain a quadratic form with respect to the $\hat{a}^{\dagger}, \hat{a}$ operators suitable for subsequent diagonalization, the following procedure might be applied. The occupation number corresponding to the state $|\mathbf{j}\rangle$ is

$$
n_{\mathbf{j}}=\left\langle\hat{a}_{\mathbf{j}}^{\dagger} \hat{a}_{\mathbf{j}}\right\rangle
$$

from which we can propose to make a substitution of operators in items with more than two of them: 


$$
\hat{a}_{\mathbf{j}}^{\dagger} \rightarrow n_{\mathbf{j}}^{x}, \quad \hat{a}_{\mathbf{j}} \rightarrow n_{\mathbf{j}}^{1-x}
$$

with a parameter $x$ to be fixed later.

After this procedure, we arrive at the diagonalization problem for the quadratic form of a general type

$$
\sum_{j, k}\left(A_{j k} \hat{a}_{j}^{\dagger} \hat{a}_{k}+B_{j k} \hat{a}_{j}^{\dagger} \hat{a}_{k}^{\dagger}+B_{j k}^{*} \hat{a}_{j} \hat{a}_{k}\right)
$$

which has been already addressed in some works [33-36].

A mathematically simpler treatment can be achieved by introducing further approximations, namely by linking the creation-annihilation operators with different indices via some factor,

$$
\hat{a}_{\mathbf{k}}=f_{\mathbf{k} \mathbf{j}} \hat{a}_{\mathbf{j}}, \quad \hat{a}_{\mathbf{k}}^{\dagger}=f_{\mathbf{k} \mathbf{j}}^{*} \hat{a}_{\mathbf{j}}^{\dagger} .
$$

With correlation (12) in mind, the following expression is consistently derived:

$$
f_{\mathbf{k j}}=\left(\frac{n_{\mathbf{k}}}{n_{\mathbf{j}}}\right)^{1-x}, \quad f_{\mathbf{k j}}^{*}=\left(\frac{n_{\mathbf{k}}}{n_{\mathbf{j}}}\right)^{x} .
$$

Provided that the occupation numbers $n_{j}$ are real and positive, so $f_{\mathbf{k j}}^{*}=f_{\mathbf{k j}}$ and thus $x=1 / 2$. For brevity, the following notation will be also used below:

$$
h_{\mathbf{j}}=n_{\mathbf{j}}^{x}=\sqrt{n_{\mathbf{j}}}
$$

Three-operator items are thus substituted in the following manner

$$
\begin{aligned}
& c_{\mathbf{j} \mathbf{k} \mathbf{l}}\left(\hat{a}_{\mathbf{j}}^{\dagger} \hat{a}_{\mathbf{k}}^{\dagger} \hat{a}_{\mathbf{l}}+\hat{a}_{\mathbf{j}}^{\dagger} \hat{a}_{\mathbf{k}} \hat{a}_{\mathbf{l}}\right) \rightarrow \frac{c_{\mathbf{j} \mathbf{k} \mathbf{l}}}{3}\left(\hat{a}_{\mathbf{j}}^{\dagger} \hat{a}_{\mathbf{k}}^{\dagger} h_{\mathbf{l}}+\hat{a}_{\mathbf{j}}^{\dagger} \hat{a}_{\mathbf{l}} h_{\mathbf{k}}+\hat{a}_{\mathbf{k}}^{\dagger} \hat{a}_{\mathbf{l}} h_{\mathbf{j}}\right)+ \\
& +\frac{c_{\mathbf{j} \mathbf{k} \mathbf{l}}}{3}\left(\hat{a}_{\mathbf{j}}^{\dagger} \hat{a}_{\mathbf{k}} h_{\mathbf{l}}+\hat{a}_{\mathbf{j}}^{\dagger} \hat{a}_{\mathbf{l}} h_{\mathbf{k}}+\hat{a}_{\mathbf{k}} \hat{a}_{\mathbf{l}} h_{\mathbf{j}}\right)
\end{aligned}
$$

while for the four-operator product one has

$$
\begin{aligned}
& c_{\mathbf{j} \mathbf{k} \mathbf{m}} \hat{a}_{\mathbf{j}}^{\dagger} \hat{a}_{\mathbf{k}}^{\dagger} \hat{a}_{\mathbf{l}} \hat{a}_{\mathbf{m}} \rightarrow \frac{c_{\mathbf{j k} \mathbf{k} \mathbf{m}}}{6}\left(\hat{a}_{\mathbf{j}}^{\dagger} \hat{a}_{\mathbf{k}}^{\dagger} h_{\mathbf{l}} h_{\mathbf{m}}+\hat{a}_{\mathbf{l}} \hat{a}_{\mathbf{m}} h_{\mathbf{j}} h_{\mathbf{k}}+\right. \\
& \left.+\hat{a}_{\mathbf{j}}^{\dagger} \hat{a}_{\mathbf{l}} h_{\mathbf{k}} h_{\mathbf{m}}+\hat{a}_{\mathbf{k}}^{\dagger} \hat{a}_{\mathbf{m}} h_{\mathbf{j}} h_{\mathbf{1}}+\hat{a}_{\mathbf{k}}^{\dagger} \hat{a}_{\mathbf{l}} h_{\mathbf{j}} h_{\mathbf{m}}+\hat{a}_{\mathbf{j}}^{\dagger} \hat{a}_{\mathbf{m}} h_{\mathbf{k}} h_{\mathbf{l}}\right) .
\end{aligned}
$$

Taking to account the symmetry of the coefficients $c_{\mathbf{j k}}$, $c_{\mathbf{j k l}}$, and $c_{\mathbf{j k l m}}$ over all the indices, after simple transformations described above the following effective Hamiltonian can be written down:

$$
\begin{aligned}
& \hat{H}_{\mathrm{eff}}=\sum_{\mathbf{j} \neq 0} \hat{a}_{\mathbf{j}}^{\dagger} \hat{a}_{\mathbf{j}}\left[\varepsilon_{\mathbf{j}}+2 N_{0} \sum_{\mathbf{k} \neq 0} f_{\mathbf{k j}} c_{\mathbf{j} \mathbf{k}}+\frac{4}{3} \sqrt{N_{0}} \sum_{\mathbf{k}, \mathbf{l} \neq 0} f_{\mathbf{k j}} c_{\mathbf{j} \mathbf{k} \mathbf{l}} h_{\mathbf{l}}+\right. \\
& \left.+\frac{1}{3} \sum_{\mathbf{k}, \mathbf{l}, \mathbf{m} \neq 0} f_{\mathbf{k j}} c_{\mathbf{j} \mathbf{k} \mathbf{l} \mathbf{m}} h_{\mathbf{l}} h_{\mathbf{m}}\right]+\sum_{\mathbf{j} \neq 0}\left(\hat{a}_{\mathbf{j}}^{\dagger} \hat{a}_{\mathbf{j}}^{\dagger}+\hat{a}_{\mathbf{j}} \hat{a}_{\mathbf{j}}\right)\left[\frac{N_{0}}{2} \sum_{\mathbf{k} \neq 0} f_{\mathbf{k j}} c_{\mathbf{j} \mathbf{k}}+\right. \\
& \left.+\frac{1}{3} \sqrt{N_{0}} \sum_{\mathbf{k}, \mathbf{l} \neq 0} f_{\mathbf{k j}} c_{\mathbf{j} \mathbf{k} \mathbf{l}} h_{\mathbf{l}}+\frac{1}{12} \sum_{\mathbf{k}, \mathbf{l}, \mathbf{m} \neq 0} f_{\mathbf{k j}} c_{\mathbf{j} \mathbf{k} \mathbf{l} \mathbf{m}} h_{\mathbf{l}} h_{\mathbf{m}}\right]
\end{aligned}
$$

The proposed transformation can be better illustrated using the Green's function formalism. Consider the equation of motion for the two-time temperature Green's function $\left\langle\left\langle\hat{a}_{q} \mid \hat{a}_{q}^{\dagger}\right\rangle\right\rangle$ :

$$
\begin{gathered}
\hbar \omega\left\langle\left\langle\hat{a}_{\mathbf{q}} \mid \hat{a}_{\mathbf{q}}^{\dagger}\right\rangle\right\rangle=\frac{1}{2 \pi}+\left\langle\left\langle\left[\hat{a}_{\mathbf{q}}, \hat{H}\right] \mid \hat{a}_{\mathbf{q}}^{\dagger}\right\rangle\right\rangle= \\
=\frac{1}{2 \pi}+\varepsilon_{\mathbf{q}}\left\langle\left\langle\hat{a}_{\mathbf{q}} \mid \hat{a}_{\mathbf{q}}^{\dagger}\right\rangle\right\rangle+N_{0} \sum_{\mathbf{k} \neq 0} c_{\mathbf{q} \mathbf{k}}\left(2\left\langle\left\langle\hat{a}_{\mathbf{k}} \mid \hat{a}_{\mathbf{q}}^{\dagger}\right\rangle\right\rangle+\left\langle\left\langle\hat{a}_{\mathbf{k}}^{\dagger} \mid \hat{a}_{\mathbf{q}}^{\dagger}\right\rangle\right\rangle\right)+ \\
+\sqrt{N_{0}} \sum_{\mathbf{k}, \mathbf{l} \neq 0} c_{\mathbf{q} \mathbf{k} \mathbf{l}}\left(2\left\langle\left\langle\hat{a}_{\mathbf{k}}^{\dagger} \hat{a}_{\mathbf{l}} \mid \hat{a}_{\mathbf{q}}^{\dagger}\right\rangle\right\rangle+\left\langle\left\langle\hat{a}_{\mathbf{k}} \hat{a}_{\mathbf{l}} \mid \hat{a}_{\mathbf{q}}^{\dagger}\right\rangle\right\rangle\right)+ \\
+\sum_{\mathbf{k}, \mathbf{l}, \mathbf{m} \neq 0} c_{\mathbf{q k} \mathbf{l} \mathbf{m}}\left\langle\left\langle\hat{a}_{\mathbf{k}}^{\dagger} \hat{a}_{\mathbf{l}} \hat{a}_{\mathbf{m}} \mid \hat{a}_{\mathbf{q}}^{\dagger}\right\rangle\right\rangle .
\end{gathered}
$$

Using the effective Hamiltonian (19) yields instead:

$$
\begin{aligned}
& \hbar \omega\left\langle\left\langle\hat{a}_{\mathbf{q}} \mid \hat{a}_{\mathbf{q}}^{\dagger}\right\rangle\right\rangle=\frac{1}{2 \pi}+\left\langle\left\langle\left[\hat{a}_{\mathbf{q}}, \hat{H}_{\mathrm{eff}}\right] \mid \hat{a}_{\mathbf{q}}^{\dagger}\right\rangle\right\rangle=\frac{1}{2 \pi}+\varepsilon_{\mathbf{q}}\left\langle\left\langle\hat{a}_{\mathbf{q}} \mid \hat{a}_{\mathbf{q}}^{\dagger}\right\rangle\right\rangle+ \\
& +N_{0} \sum_{\mathbf{k} \neq 0} c_{\mathbf{q} \mathbf{k}}\left(2 f_{\mathbf{k q}}\left\langle\left\langle\hat{a}_{\mathbf{q}} \mid \hat{a}_{\mathbf{q}}^{\dagger}\right\rangle\right\rangle+f_{\mathbf{k q}}\left\langle\left\langle\hat{a}_{\mathbf{q}}^{\dagger} \mid \hat{a}_{\mathbf{q}}^{\dagger}\right\rangle\right)+\right. \\
& +\frac{2}{3} \sqrt{N_{0}} \sum_{\mathbf{k}, \mathbf{l} \neq 0} c_{\mathbf{q} \mathbf{k} \mathbf{l}}\left(2 f_{\mathbf{k q}} h_{\mathbf{l}}\left\langle\left\langle\hat{a}_{\mathbf{q}} \mid \hat{a}_{\mathbf{q}}^{\dagger}\right\rangle\right\rangle+f_{\mathbf{k q}} h_{\mathbf{l}}\left\langle\left\langle\hat{a}_{\mathbf{q}}^{\dagger} \mid \hat{a}_{\mathbf{q}}^{\dagger}\right\rangle\right\rangle\right)+ \\
& +\frac{1}{12} \sum_{\mathbf{k}, \mathbf{l}, \mathbf{m} \neq 0} c_{\mathbf{q} \mathbf{k} \mathbf{l} \mathbf{m}} f_{\mathbf{k q}} h_{\mathbf{l}} h_{\mathbf{m}}\left(\left\langle\left\langle\hat{a}_{\mathbf{q}}^{\dagger} \mid \hat{a}_{\mathbf{q}}^{\dagger}\right\rangle\right\rangle+\left\langle\left\langle\hat{a}_{\mathbf{q}} \mid \hat{a}_{\mathbf{q}}^{\dagger}\right\rangle\right\rangle\right) .
\end{aligned}
$$

The proposed operator substitutions would thus lead to a closed set of two equations of motion (for $\left\langle\left\langle\hat{a}_{\mathbf{q}} \mid \hat{a}_{\mathbf{q}}^{\dagger}\right\rangle\right\rangle$ and $\left.\left\langle\left\langle\hat{a}_{\mathbf{q}}^{\dagger} \mid \hat{a}_{\mathbf{q}}^{\dagger}\right\rangle\right\rangle\right)$ by means of getting rid of the off-diagonal functions

$$
\sum_{\mathbf{k} \neq 0} c_{\mathbf{q} \mathbf{k}}\left\langle\left\langle\hat{a}_{\mathbf{k}} \mid \hat{a}_{\mathbf{q}}^{\dagger}\right\rangle\right\rangle \rightarrow\left\langle\left\langle\hat{a}_{\mathbf{q}} \mid \hat{a}_{\mathbf{q}}^{\dagger}\right\rangle\right\rangle \sum_{\mathbf{k} \neq 0} c_{\mathbf{q} \mathbf{k}} f_{\mathbf{k q}} \equiv G_{0}(\mathbf{q})\left\langle\left\langle\hat{a}_{\mathbf{q}} \mid \hat{a}_{\mathbf{q}}^{\dagger}\right\rangle\right\rangle
$$

as well as decoupling higher-order functions

$$
\begin{aligned}
\sum_{\mathbf{k}, \mathbf{l} \neq 0} c_{\mathbf{q} \mathbf{k} \mathbf{l}} & \left\langle\left\langle\hat{a}_{\mathbf{k}}^{\dagger} \hat{a}_{\mathbf{l}} \mid \hat{a}_{\mathbf{q}}^{\dagger}\right\rangle\right\rangle \rightarrow G_{\mathbf{l}}(\mathbf{q})\left\langle\left\langle\hat{a}_{\mathbf{q}} \mid \hat{a}_{\mathbf{q}}^{\dagger}\right\rangle\right\rangle+ \\
+ & G_{2}(\mathbf{q})\left\langle\left\langle\hat{a}_{\mathbf{q}}^{\dagger} \mid \hat{a}_{\mathbf{q}}^{\dagger}\right\rangle\right\rangle, \text { etc. }
\end{aligned}
$$

with coefficients $G_{1,2}(\mathbf{q})$ linked to $f_{\mathbf{k q}}$ and $h_{\mathbf{l}}$. The former relation can be - to a certain extent - related to the socalled quantum-number conservation condition [26]

$$
\left\langle\hat{a}_{\mathbf{k}}^{\dagger} \hat{a}_{\mathbf{q}}\right\rangle=\delta_{\mathbf{k}, \mathbf{q}}\left\langle\hat{a}_{\mathbf{q}}^{\dagger} \hat{a}_{\mathbf{q}}\right\rangle,
$$

while the latter is a mean-field-like approximation. A similar approach proved to be successful in modeling the spectrum of strongly interacting Bose-systems [37].

The following notations will be used below:

$$
\begin{gathered}
\gamma_{\mathbf{j}}=\frac{N_{0}}{2} \sum_{\mathbf{k} \neq 0} f_{\mathbf{k j}} c_{\mathbf{j} \mathbf{k}} ; \\
\eta_{\mathbf{j}}=\frac{1}{3} \sqrt{N_{0}} \sum_{\mathbf{k}, \mathbf{l} \neq 0} f_{\mathbf{k j}} c_{\mathbf{j} \mathbf{k} \mathbf{l}} h_{\mathbf{l}} ;
\end{gathered}
$$




$$
\varphi_{\mathbf{j}}=\frac{1}{12} \sum_{\mathbf{k}, \mathbf{l}, \mathbf{m} \neq 0} f_{\mathbf{k j}} c_{\mathbf{j k l m}} h_{\mathbf{l}} h_{\mathbf{m}}
$$

The Hamiltonian is thus the quadratic form

$$
\hat{H}_{\mathrm{eff}}=\sum_{\mathbf{j} \neq 0}\left\{\left(\varepsilon_{\mathbf{j}}+4 Q_{\mathbf{j}}\right) \hat{a}_{\mathbf{j}}^{\dagger} \hat{a}_{\mathbf{j}}+Q_{\mathbf{j}}\left(\hat{a}_{\mathbf{j}}^{\dagger} \hat{a}_{\mathbf{j}}^{\dagger}+\hat{a}_{\mathbf{j}} \hat{a}_{\mathbf{j}}\right)\right\}
$$

with

$$
Q_{\mathbf{j}}=\gamma_{\mathbf{j}}+\eta_{\mathbf{j}}+\varphi_{\mathbf{j}}
$$

which can be diagonalized using the standard Bogoliubov's $u-v$ transform.

The operators in each mode (state) are expressed as

$$
\hat{a}_{\mathbf{j}}=u_{\mathbf{j}} \hat{b}_{\mathbf{j}}+v_{\mathbf{j}} \hat{b}_{\mathbf{j}}^{\dagger}, \quad \hat{a}_{\mathbf{j}}^{\dagger}=u_{\mathbf{j}}^{*} \hat{b}_{\mathbf{j}}^{\dagger}+v_{\mathbf{j}}^{*} \hat{b}_{\mathbf{j}}
$$

From the bosonic commutator

$$
\left[\hat{a}_{\mathbf{j}}, \hat{a}_{\mathbf{j}}^{\dagger}\right]=1
$$

requiring that off-diagonal products $\hat{b}_{\mathbf{j}} \hat{b}_{\mathbf{j}}$ and $\hat{b}_{\mathbf{j}}^{\dagger} \hat{b}_{\mathbf{j}}^{\dagger}$ are eliminated in the final expressions, the Hamiltonian diagonalizes to the form

$$
\hat{H}_{\mathrm{eff}}=\sum_{\mathbf{j} \neq 0} E_{\mathbf{j}} \hat{b}_{\mathbf{j}}^{\dagger} \hat{b}_{\mathbf{j}},
$$

where the elementary excitation spectrum is

$$
E_{\mathbf{j}}=\sqrt{\varepsilon_{\mathbf{j}}^{2}+8 \varepsilon_{\mathbf{j}} Q_{\mathbf{j}}+12 Q_{\mathbf{j}}^{2}} .
$$

Evaluation of the above expression cannot be done without specifying the functional dependence of $f_{\mathbf{j}}$ and $h_{\mathbf{j}}$. On the other hand, the $c$ coefficients (matrix elements of the potential energy operator) can be rewritten as follows:

$$
\begin{gathered}
c_{\mathbf{j k}}=c_{j_{1} k_{1}} \ldots c_{j_{D} k_{D}}, \quad c_{\mathbf{j k l}}=c_{j_{1} k_{1} l_{1}} \ldots c_{j_{D} k_{D} l_{D}}, \\
c_{\mathbf{j k \mathbf { m }}}=c_{j_{1} k_{1} l_{1} m_{1}} \ldots c_{j_{D}{ }^{k}{ }{ }{ }^{m} D}
\end{gathered}
$$

because the $D$-dimensional harmonic oscillator eigenfunctions are factorized into the one-dimensional ones.

\section{A one-dimensional problem}

To provide an example of calculations with the suggested effective Hamiltonian, a one-dimensional problem can be considered. For the harmonic oscillator of mass $m$ and frequency $\omega$, the eigenfunctions are

$$
\begin{gathered}
|j\rangle=\frac{1}{\sqrt{2^{j} j !}}\left(\frac{m \omega}{\pi \hbar}\right)^{1 / 4} \mathrm{e}^{-m \omega x^{2} / 2 \hbar} H_{j}\left(\sqrt{\frac{m \omega}{\hbar} x}\right), \\
j=0,1,2, \ldots,
\end{gathered}
$$

where $H_{j}(\xi)$ is the Hermite polynomial.

Using the harmonic oscillator length

$$
a_{\mathrm{ho}}=\sqrt{\frac{\hbar}{m \omega}}
$$

matrix elements (8)-(10) are obtained in the form

$$
\begin{aligned}
c_{j k} & =\frac{g}{a_{\mathrm{ho}}} \frac{1}{\sqrt{2^{j+k} j ! k !}} \frac{1}{\pi} \int_{-\infty}^{\infty} \mathrm{e}^{-2 \xi^{2}} H_{j}(\xi) H_{k}(\xi) d \xi= \\
& =\frac{g}{a_{\mathrm{ho}}} \frac{1}{\pi \sqrt{2}} \frac{(-1)^{(j-k) / 2}}{\sqrt{j ! k !}} \Gamma\left(\frac{j+k+1}{2}\right) .
\end{aligned}
$$

$$
\begin{gathered}
c_{j k l}=\frac{g}{a_{\mathrm{ho}}} \frac{1}{\sqrt{2^{j+k+l} j ! k ! l !}} \frac{1}{\pi} \int_{-\infty}^{\infty} \mathrm{e}^{-2 \xi^{2}} H_{j}(\xi) H_{k}(\xi) H_{l}(\xi) d \xi= \\
=\frac{g}{a_{\mathrm{ho}}} \frac{1}{\pi^{2} \sqrt{2}} \frac{1}{\sqrt{j ! k ! l !}} \Gamma\left(\frac{j+k-l+1}{2}\right) \Gamma\left(\frac{j-k+l+1}{2}\right) \times \\
\times \Gamma\left(\frac{k-j+l+1}{2}\right),
\end{gathered}
$$

$$
c_{j k l m}=\frac{g}{a_{\mathrm{ho}}} \frac{1}{\sqrt{2^{j+k+l+m} j ! k ! ! ! m !}} \frac{1}{\pi} \times
$$

$$
\times \int_{-\infty}^{\infty} \mathrm{e}^{-2 \xi^{2}} H_{j}(\xi) H_{k}(\xi) H_{l}(\xi) H_{m}(\xi) d \xi=
$$

$$
=\frac{g}{a_{\mathrm{ho}}} \frac{1}{\pi \sqrt{2}} \frac{(-1)^{(k-j+m-l) / 2}}{\sqrt{j ! k ! l ! m !}} \times
$$$$
\times \frac{\Gamma\left(\frac{j+k+l-m+1}{2}\right) \Gamma\left(\frac{j-k+l+m+1}{2}\right)}{\Gamma\left(\frac{j-k+l-m+1}{2}\right)} \times
$$

$$
\times{ }_{3} F_{2}\left(\begin{array}{c}
-j, \quad-l, \frac{k-j+m-l+1}{2} ; \\
\frac{m-j-k-l+1}{2}, \frac{k-j-l-m+1}{2} ;
\end{array}\right) .
$$

The integration can be made in a closed form as discussed, for instance, in [5,38-40]. In the above expressions, $\Gamma(z)$ is Euler's gamma-function, and ${ }_{3} F_{2}\left(a_{1}, a_{2}, a_{3} ; b_{1}, b_{2} ; z\right)$ is the hypergeometric function. Note that indices in the $c$ coefficients $(j+k, j+k+l$, and $j+k+l+m)$ must sum up to an even number otherwise the respective integrals are zero due to the parity property of the Hermite polynomial products.

Models for $f_{k j}$ and $h_{j}$ use the occupation numbers $n_{j}$ :

$$
f_{k j}=\sqrt{\frac{n_{k}}{n_{j}}}, \quad h_{j}=\sqrt{n_{j}} .
$$

Generally, for harmonic oscillators

$$
n_{j}=\frac{1}{z^{-1} \mathrm{e}^{\hbar \omega j / T}-1}
$$


and fugacity $z$ can be approximately put unity at low temperatures corresponding to the BEC regime.

In the limit of $\hbar \omega>>T$,

$$
n_{j} \simeq \mathrm{e}^{-\hbar \omega j / T} .
$$

In most experiments with cold atomic gases an opposite condition is satisfied, $\hbar \omega<<T$, cf. $[3,41]$. It leads to

$$
n_{j} \simeq \frac{T}{\hbar \omega j} \text {. }
$$

With this simple expression we will make numerical calculations below. Dependences of $f_{k j}$ and $h_{j}$ become thus:

$$
f_{k j}=\sqrt{\frac{j}{k}}, \quad h_{j}=\sqrt{\frac{T}{\hbar \omega j}} .
$$

This means in particular that there will be no temperature dependence in $\gamma_{j}$ but corrections $\eta_{j}$ and $\varphi_{j}$ are proportional to $\sqrt{T}$ and $T$, respectively.

At BEC, the occupation of the lowest level $N_{0}$ approaches the total number of particles $N$, so we put $N_{0}=N$ is expressions for $\gamma_{j}$ and $\eta_{j}$ yielding

$$
\begin{gathered}
\gamma_{j}=\frac{g N}{a_{\mathrm{ho}}} \bar{\gamma}_{j}, \\
\eta_{j}=\frac{g}{a_{\mathrm{ho}}} \sqrt{\frac{N T}{\hbar \omega}} \bar{\eta}_{j}, \\
\varphi_{j}=\frac{g}{a_{\mathrm{ho}}} \frac{T}{\hbar \omega} \bar{\varphi}_{j},
\end{gathered}
$$

where $\bar{\gamma}_{j}, \bar{\eta}_{j}$, and $\bar{\varphi}_{j}$ are dimensionless coefficients obtained by inserting Eqs. (32)-(34) with $f_{k j}$ and $h_{j}$ from (35) into one-dimensional analogs of Eqs. (20)-(22).

Spectrum (28) in one dimension with $\varepsilon_{j}=\hbar \omega j$ becomes

$$
E_{j}=\hbar \omega j \sqrt{1+8 \frac{\gamma_{j}+\eta_{j}+\varphi_{j}}{\hbar \omega j}+12\left(\frac{\gamma_{j}+\eta_{j}+\varphi_{j}}{\hbar \omega j}\right)^{2}} .
$$

Upon introducing dimensionless parameters,

$$
x=\frac{g N}{a_{\mathrm{ho}}} \frac{1}{\hbar \omega}, \quad y=\frac{T}{\hbar \omega N},
$$

the spectrum yields

$$
\begin{gathered}
E_{j}= \\
=\hbar \omega j \sqrt{1+8 \frac{x \bar{\gamma}_{j}+x \sqrt{y} \bar{\eta}_{j}+y \bar{\varphi}_{j}}{j}+12\left(\frac{x \bar{\gamma}_{j}+x \sqrt{y} \bar{\eta}_{j}+y \bar{\varphi}_{j}}{j}\right)^{2}} .
\end{gathered}
$$

Numerical values of $\bar{\gamma}_{j}, \bar{\eta}_{j}$, and $\bar{\varphi}_{j}$ are shown in Table 1 . Dependences of the energies of the first three levels $j=1,2,3$ on the values of $x$ and $y$ parameters are shown in Fig. 1.
Table 1. Values of dimensionless coefficients in Eqs. (40)-(42) depending on the level number $j$

\begin{tabular}{c|c|c|c}
\hline \hline$j$ & $\bar{\gamma}_{j}$ & $\bar{\eta}_{j}$ & $\bar{\varphi}_{j}$ \\
\hline \hline 1 & 0,074987 & 0,020906 & 0,041237 \\
2 & 0,049719 & 0,047775 & 0,073939 \\
3 & $-0,067169$ & 0,045062 & 0,087680 \\
4 & $-0,044015$ & $-0,022696$ & 0,081353 \\
5 & 0,041606 & $-0,044378$ & 0,056191 \\
6 & 0,030211 & 0,005830 & 0,036522 \\
7 & $-0,023169$ & 0,030029 & 0,039952 \\
8 & $-0,018589$ & 0,000509 & 0,049381 \\
9 & 0,012314 & $-0,017838$ & 0,045257 \\
10 & 0,010780 & $-0,001987$ & 0,035160 \\
11 & $-0,006394$ & 0,009961 & 0,033071 \\
12 & $-0,006034$ & 0,001827 & 0,036096 \\
13 & 0.003288 & $-0,005385$ & 0,031684 \\
14 & 0,003311 & $-0,001339$ & 0,031684 \\
15 & $-0,001702$ & 0,002872 & 0,029970 \\
16 & $-0,001815$ & 0,000955 & 0,030215 \\
17 & 0,000929 & $-0,001549$ & 0,029776 \\
18 & 0.001035 & $-0,000784$ & 0,028471 \\
19 & $-0,000590$ & 0,000883 & 0,027376 \\
20 & $-0,000678$ & 0,000844 & 0,026641 \\
25 & 0,000843 & $-0,000360$ & 0,023545 \\
30 & 0,001743 & $-0,004430$ & 0,023388 \\
50 & 0,007818 & 0,000009 & 0,025675 \\
100 & $-0,000160$ & 0,000125 & 0,000214 \\
\hline \hline & & &
\end{tabular}

Note that when $x$ reaches values above 2, an intersection of the energies $E_{2}$ and $E_{3}$ occurs. A similar behavior is possible for some other levels since $\bar{\gamma}_{j}$ and $\bar{\eta}_{j}$ can have both positive and negative values.

Obviously, the validity of the approximation decreases for items containing $\eta_{j}$ and even more for $\varphi_{j}$ terms because of number of simplifications applied, in comparison with the $\gamma_{j}$ term.

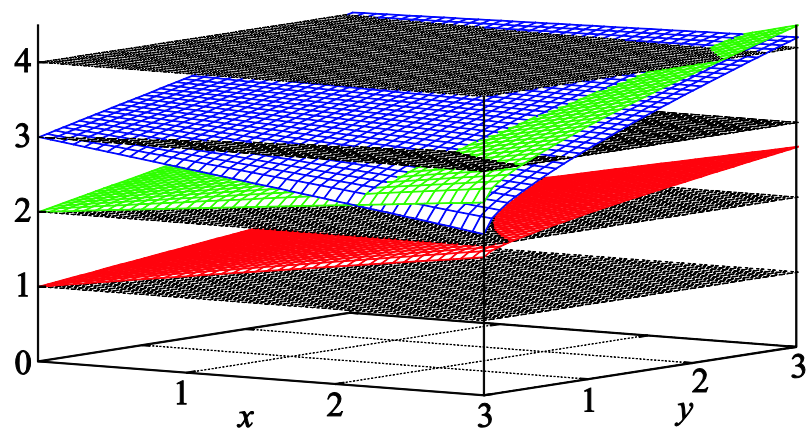

Fig. 1. (Color online) Energies of the first three excited levels $E_{1}, E_{2}, E_{3}$ (vertical axis, in $\hbar \omega$ units) given by Eq. (45) as functions of the $x$ and $y$ parameters (44). Red surface is $E_{1}$, green sufrace is $E_{2}$, and blue surface is $E_{3}$. Grey-shaded planes correspond to noninteracting oscillator energies $\varepsilon_{1}, \ldots, \varepsilon_{4}$. 


\section{A binary Bose mixture}

Weakly-interacting Bose mixtures confined in harmonic traps became a subject of experimental and theoretical studies recently, e. g., mixture of ytterbium bosonic isotopes $[42,43]$ or rubidium with some other elements [44], see also [45].

Let the system contain two boson species, $a$ and $b$, being characterized by coupling constants $g_{a}, g_{b}$, and $g_{a b}$, the latter corresponds to the inter-species interaction. The one particle problems remain the same for each species:

$$
\hat{H}_{1 a}=\hat{H}_{0 a}+U_{a} ; \quad \hat{H}_{1 b}=\hat{H}_{0 b}+U_{b},
$$

where the non-interacting oscillator Hamiltonians are

$$
\hat{H}_{0 a}=\hat{K}_{a}+V_{a} ; \quad \hat{H}_{0 b}=\hat{K}_{b}+V_{b}
$$

with trapping potentials

$$
\begin{aligned}
& V_{a}(\mathbf{x})=\frac{m_{a}}{2}\left(\omega_{1}^{2} x_{1}^{2}+\ldots+\omega_{D}^{2} x_{D}^{2}\right) \\
& V_{b}(\mathbf{x})=\frac{m_{b}}{2}\left(\omega_{1}^{2} x_{1}^{2}+\ldots+\omega_{D}^{2} x_{D}^{2}\right)
\end{aligned}
$$

and the interaction items are

$$
U_{a}=g_{a} \delta(\mathbf{x}) ; \quad U_{b}=g_{b} \delta(\mathbf{x}) ; \quad U_{a b}=g_{a b} \delta(\mathbf{x}) .
$$

In the occupation number formalism we obtain:

$$
\begin{gathered}
\hat{H}=\sum \hat{H}_{1 a}+\sum \hat{H}_{1 b}+\sum U_{a b}= \\
=\sum_{n_{a}} \varepsilon_{n_{a}} \hat{a}_{n_{a}}^{\dagger} \hat{a}_{n_{a}}+\sum_{n_{a}} \varepsilon_{n_{b}} \hat{b}_{n_{b}}^{\dagger} \hat{b}_{n_{b}}+U,
\end{gathered}
$$

where the unidexed sums run over all the relevant particles and $U$ is the total potential energy in the system. The following Hamiltonian is thus of special interest:

$$
\begin{gathered}
\hat{H}^{\prime}=\sum_{m n}\left(A_{m n} \hat{a}_{m}^{\dagger} \hat{a}_{n}+B_{m n} \hat{b}_{m}^{\dagger} \hat{b}_{n}+C_{m n} \hat{a}_{m}^{\dagger} \hat{a}_{n}^{\dagger}+C_{m n}^{*} \hat{a}_{m} \hat{a}_{n}+\right. \\
+D_{m n} \hat{b}_{m}^{\dagger} \hat{b}_{n}^{\dagger}+D_{m n}^{*} \hat{b}_{m} \hat{b}_{n}+F_{m n} \hat{a}_{m}^{\dagger} \hat{b}_{n}^{\dagger}+F_{m n}^{*} \hat{a}_{m} \hat{b}_{n}+ \\
\left.+G_{m n} \hat{a}_{m}^{\dagger} \hat{b}_{n}+G_{m n}^{*} \hat{b}_{m}^{\dagger} \hat{a}_{n}\right)
\end{gathered}
$$

which is obtained by getting rid of three- and fouroperator products as described in Sec. 3. A diagonalized Hamiltonian

$$
H^{\prime \prime}=\sum_{j} E_{j} \hat{\xi}_{j}^{\dagger} \hat{\xi}_{j}=\sum_{m} E_{m}^{(\alpha)} \hat{\alpha}_{m}^{\dagger} \hat{\alpha}_{m}+\sum_{n} E_{n}^{(\beta)} \hat{\beta}_{n}^{\dagger} \hat{\beta}_{n}
$$

can be obtained by a direct application of the diagonalization procedure similar to that discussed in papers [33-36]. It is technically complicated and involves nonlinear equations for infinite-size matrices; a perturbative solution might be a good alternative.

On the other hand, the procedure from Sec. 3 can be applied leading to

$$
\hat{H}^{\prime \prime}=\sum_{j} \hat{h}_{j}
$$

where the diagonalization of the quadratic form in each mode $\hat{h}_{j}$ is nothing but the problem reducing to a biquadratic equation.

For a simplified expression representing a single mode

$$
\begin{gathered}
\hat{h}=\varepsilon_{a} \hat{a}^{\dagger} \hat{a}+\varepsilon_{b} \hat{b}^{\dagger} \hat{b}+ \\
+\lambda_{a}\left(4 \hat{a}^{\dagger} \hat{a}+\hat{a}^{\dagger} \hat{a}^{\dagger}+\hat{a} \hat{a}\right)+\lambda_{b}\left(4 \hat{b}^{\dagger} \hat{b}+\hat{b}^{\dagger} \hat{b}^{\dagger}+\hat{b} \hat{b}\right)+ \\
+\lambda_{a b}\left(\hat{a}^{\dagger} \hat{b}+\hat{a} \hat{b}^{\dagger}\right),
\end{gathered}
$$

where $\lambda_{a}, \lambda_{b}$, and $\lambda_{a b}$ are linked to interaction parameters $g_{a}, g_{b}$, and $g_{a b}$ as well as contain some effective contributions from simplifications of the original Hamiltonian, one can obtain easily two branches of the excitation spectrum:

$$
E^{(1,2)}=\sqrt{p / 2 \pm \sqrt{p^{2} / 4-q}}
$$

with

$$
p=\left(\varepsilon_{a}+4 \lambda_{a}\right)^{2}+\left(\varepsilon_{b}+4 \lambda_{b}\right)^{2}-\lambda_{a}^{2}-\lambda_{b}^{2}+2 \lambda_{a b}^{2}
$$

and

$$
\begin{gathered}
q=\left(\varepsilon_{a}+4 \lambda_{a}\right)^{2}\left(\varepsilon_{a}+4 \lambda_{a}\right)^{2}- \\
-\lambda_{a}^{2}\left(\varepsilon_{b}+4 \lambda_{b}\right)^{2}-\lambda_{b}^{2}\left(\varepsilon_{a}+4 \lambda_{a}\right)^{2}-\lambda_{a}^{2} \lambda_{b}^{2}- \\
-2 \lambda_{a b}^{2}\left(\varepsilon_{a}+4 \lambda_{a}\right)\left(\varepsilon_{b}+4 \lambda_{b}\right)-2 \lambda_{a} \lambda_{b} \lambda_{a b}^{2}-\lambda_{a b}^{4} .
\end{gathered}
$$

\section{Discussion}

In summary, an approach was proposed for obtaining an effective Hamiltonian for bosonic systems in a harmonic trap. An approximation consisting in the substitution of the off-diagonal creation-annihilation operator products, $\hat{a}_{\mathbf{k}}^{\dagger} \hat{a}_{\mathbf{j}} \rightarrow f_{\mathbf{k j}} \hat{a}_{\mathbf{j}}^{\dagger} \hat{a}_{\mathbf{j}}$ and alike, gave the possibility to diagonalize the Hamiltonian in a mathematically simple fashion and to calculate the elementary excitation spectrum. The factors $f_{\mathbf{k j}}$ were related to the occupation numbers. Other models, beyond a simple dependence in the $T>\hbar_{\omega}$ limit considered here, could be tested in subsequent works.

A more consistent approach to derive the expressions for $h_{\mathbf{j}}$ and $f_{\mathbf{k j}}$ would require solving a variational problem similar to the Gross-Pitaevskii one but being of a higher order. The most straightforward way is to make a decomposition of the operator $\hat{a}$ into a non-operator part $h$ and a small correction $\hat{\alpha}$, that is $\hat{a}=h+\hat{\alpha}$. It was not addressed in this paper due to significant mathematical complications, which were in contradiction with the idea of intended effective simplification of the diagonalization problem. Such a systematic analysis requires a separate study, while the results of the present paper allow for making computations with different expressions for $h_{\mathbf{j}}$ and $f_{\mathbf{k j}}$. 
Numerical calculations of the excitation spectrum for the one-dimensional systems will be further extended to higher-dimensional systems as all the analytical expressions were derived in this paper. Binary bosonic mixtures might be also treated using the proposed approach.

The obtained excitation spectrum makes it possible to calculate the properties of the system, like energy, specific heat, and condensate fraction, using standard thermodynamic methods [33]. Carrying out such computations is more a technical task and requires specification of the system parameters, including the trap geometry for two- or three-dimensional systems.

I am grateful to the anonymous Referees for useful comments and criticisms, which open a possibility to develop the method of the present paper on a more rigorous level.

The paper is based on the research provided by the grant support of the State Fund For Fundamental Research of Ukraine, Project F-64/41-2015 (No. 0115U004838).

1. M.H. Anderson, J.N. Ensher, M.R. Matthews, C.E. Wieman, and E.A. Cornell, Science 269, 198 (1995).

2. K.B. Davis, M. Mewes, M.R. Andrews, N.J. van Druten, D.S. Durfee, D.M. Kurn, and W. Ketterle, Phys. Rev. Lett. 75, 3969 (1995).

3. D.S. Jin, J.R. Ensher, M.R. Matthews, C.E. Wieman, and E.A. Cornell, Phys. Rev. Lett. 77, 420 (1996).

4. S. Stringari, Phys. Rev. Lett. 77, 2360 (1996).

5. L. You, W. Hoston, M. Lewenstein, and M. Marinescu, Acta Phys. Polon. A 93, 211 (1998).

6. S. Grossmann and M. Holthaus, Phys. Lett. A 208, 188 (1995).

7. H. Haugerud, T. Haugset, and F. Ravndal, Phys. Lett. A 225, 18 (1997).

8. W.J. Mullin, J. Low Temp. Phys. 106, 615 (1997).

9. F. Gerbier, Europhys. Lett. 66, 771 (2004).

10. A.S. Parkins and D.F. Walls, Phys. Rep. 303, 1 (1998).

11. F. Dalfovo, S. Giorgini, L.P. Pitaevskii, and S. Stringari, Rev. Mod. Phys. 71, 463 (1999).

12. I. Bloch, J. Dalibard, and W. Zwerger, Rev. Mod. Phys. 80, 885 (2008).

13. V.S. Bagnato, D.J. Frantzeskakis, P.G. Kevrekidis, B.A. Malomed, and D. Mihalache, Rom. Rep. Phys. 67, 5 (2015).

14. C. Menotti and S. Stringari, Phys. Rev. A 66, 043610 (2002).

15. Y.-W. Luo and J.-S. Chen, Commun. Theor. Phys. 60, 673 (2013).

16. K.R.A. Hazzard and E.J. Mueller, Phys. Rev. A 76, 063612 (2007)
17. M. Yamashita, S. Kato, A. Yamaguchi, S. Sugawa, T. Fukuhara, S. Uetake, and Y. Takahashi, Phys. Rev. A 87, 041604(R) (2013)

18. I.V. Stasyuk, O.V. Velychko, and I.R. Dulepa, Ukr. J. Phys. 59, 993 (2014).

19. N.N. Bogoliubov, J. Phys. USSR 11, 23 (1947).

20. P. Grech and R. Seiringer, Commun. Math. Phys. 322, 559 (2013).

21. R. Seiringer, J. Math. Phys. 55, 075209 (2014).

22. C. Trallero-Giner, M.V. Durnev, Y. Núñez Fernández, M.I. Vasilevskiy, V. López-Richard, and A. Kavokin, Phys. Rev. $B$ 89, 205317 (2014).

23. V.I. Yukalov and H. Kleinert, Phys. Rev. A 73, 063612 (2006).

24. T. Ernst, T. Paul, and P. Schlagheck, Phys. Rev. A 81, 013631 (2010).

25. V.I. Yukalov, E.P. Yukalova, and V.S. Bagnato, Laser Phys. 19, 686 (2009).

26. V.I. Yukalov, Phys. Part. Nucl. 42, 460 (2011).

27. N. Argaman and Y.B. Band, Phys. Rev. A 83, 023612 (2011).

28. A. Alastuey and J. Piasecki, Phys. Rev. E 84, 041122 (2011).

29. H. Kim, C.S. Kim, C.L. Huang, H.-S. Song, and X.-X. Yi, Phys. Rev. A 85, 033611 (2012).

30. H. Kim, C.-S. Kim, C.-L. Huang, H.-S. Song, and X.-X. Yi, Phys. Rev. A 85, 053629 (2012).

31. A. Roy and D. Angom, Phys. Rev. A 92, 011601 (2015).

32. X.-L. Chen, Y. Li, and H. Hu, Phys. Rev. A 91, 063631 (2015).

33. A.A. Rovenchak, J. Low Temp. Phys. 148, 411 (2007).

34. J.H.P. Colpa, Physica A 93, 327 (1978).

35. C. Tsallis, J. Math. Phys. 19, 277 (1978).

36. M. Marinescu and A.F. Starace, Phys. Rev. A 56, 570 (1997).

37. A. Rovenchak, Z. Naturforsch. A 70, 73 (2015).

38. E.C. Titchmarsh, J. London Math. Soc. s1-23, 15 (1949).

39. R.D. Lord, J. London Math. Soc. s1-24, 101 (1949).

40. S.D. Bajpai, Ann. Math. Siles. 6, 13 (1992).

41. S. Giorgini, L.P. Pitaevskii, and S. Stringari, Phys. Rev. A 54, R4633 (1996).

42. K. Kasamatsu and M. Tsubota, J. Low Temp. Phys. 150, 599 (2008).

43. R. Campbell, G.-L. Oppo, and M. Borkowski, Phys. Rev. E 91, 012909 (2015).

44. B. Pasquiou, A. Bayerle, S.M. Tzanova, S. Stellmer, J. Szczepkowski, M. Parigger, R. Grimm, and F. Schreck, Phys. Rev. A 88, 023601 (2013).

45. B. Van Schaeybroeck, Physica A 392, 3806 (2013). 